\title{
Introduction to the special issue: quantifying collectivity
}

\author{
Bryan C. Daniels ${ }^{2} \cdot$ Manfred D. Laubichler $^{2}$. Jessica C. Flack ${ }^{1}$
}

Received: 26 October 2021 / Accepted: 28 October 2021 / Published online: 17 November 2021

(c) The Author(s), under exclusive licence to Springer-Verlag GmbH Germany, part of Springer Nature 2021

Biological systems are diverse, ranging from tightly packed, highly integrated, many-body systems like eukaryotic cells to decentralized microbial biofilms, to relatively small primate groups with on the order of 100 behaviorally flexible individuals all the way to large, complex societies (both insects and human) and ecosystems. This range suggests there is variation in both how collective a system is as well as how it is collective.

Yet we still know little about this diversity in collectivity, how to quantify it, or what its implications are for system robustness, evolvability, or adaptive output. Part of the reason we know so little about the nature of collectivity is that (ironically) we tend to study aspects of collectivity in isolation. Using the tools of dynamical systems, researchers have asked how fireflies synchronize flashes (e.g., RamírezÁvila et al. 2019). Using the tools of statistical physics, researchers have asked how locusts coordinate movement (e.g., Buhl et al. 2006), how bird flocks choose a trajectory (e.g., Bialek et al. 2012) and what role noise plays in inducing transitions between ordered and disordered collective states (e.g., Jhawar et al. 2020). Disciplinary traditions and a predilection for mathematical tractability have translated into the collective behavior community collectively ignoring growing evidence for microscopic complexity: as a relatively simple example, consider that when male fireflies, during mating season, synchronize their flashes they are also engaging in collective motion but their syncing is typically studied independently of their swarming. O'Keeffe, Hong, and Strogatz (O'Keeffe et al. 2017) in a recent paper made this point to motivate the development of their hybrid swarmalator model-a step forward in bridging the sync and swarm divide and a step in studying how different modalities of collective behavior influence each other. Although the field

Jessica C. Flack

jflack@santafe.edu

Santa Fe Institute, Santa Fe, USA

2 School of Complex Adaptive Systems, Arizona State University, Tempe, USA of collective behavior is seeing progress on these issues in, for example, the work of Peleg and collaborators (e.g., Sarfati et al. 2021) on self-organization and synchronization in firefly swarms and in the work of Cavagna and collaborators (Cavagna et al. 2017) on dynamical scaling in midge swarms, these types of studies are not sufficient. To understand the implications of collectivity for group performance, robustness, and evolvability, we need to better know what we mean by collectivity. And for that we need a formalism for quantifying it and the conceptual structures that go with such a formalism.

What might such a formalism look like? Perhaps the closest attempts to develop a formalism of the sort we have in mind can be found in maximum entropy applications and the study of individuality. In the case of hierarchical maximum entropy models used in the study of collective behavior, a goal has been to determine the dominant order of interactions (e.g., pairwise, higher order) that can best account for a macroscopic observable (see, for example, Mora et al. 2010). To ask the question, "What order of interaction dominates?" is to recognize that systems might vary in their degree of decomposability.

Within the context of individuality, Krakauer et al. in recent work (Krakauer et al. 2020) created a first principles taxonomy of individuality called the Information Theory of Individuality or ITI. The formalism, which operationalizes individuality as temporal uncertainty reduction reframes individuality as a dynamical process thereby not excluding a priori, for example, social systems and slime molds simply because they are not spatially cohesive or don't replicate in the favored sense. The ITI allows for different degrees of individuality and also types of individuality, depending on whether information is "stored" in the environment, the entity's past, or some interaction of the two. The development of the ITI implies a conceptual reorientation, away from a metaphysical commitment to stable (and generally static) entities toward a dynamic and process-focused conception, where the process itself defines the relevant entities and objects of analysis. 
The individuality example also illustrates that a concept thought to have many dimensions (e.g., replication, boundedness, component alignment of interests or correlated fitness, etc.) can in fact be distilled into a relatively simple first principles-based, formal definition. It is important to recall, however, that individuality has long been debated as a concept in the biological and philosophical literatures and the ITI formalism, informed by that noisy and largely qualitative debate, can be understood as a reaction to it-a desire to make progress by being formal and precise. The study of collective behavior has proceeded very differently. It has been quantitative from the start but the focus, as discussed above, has been on using quantitative methods to study different aspects of collectivity, like syncing or swarming, in isolation. The concept of collectivity-what it is and how it varies across systems-has never really been the center of attention.

But in both cases-individuality and collectivity-the vast majority of research started with a pre-conceived notion of what the relevant entities are-a school of fish, a group of primates, a flock of birds or a cell, an organism, a colony, etc. - and then stared to analyze isolated properties of these entities (Krakauer et al. 2020). By focusing on a formalism that describes functional, dynamical, and relational properties in a rigorous way, we reverse the order of questions. This allows us to (1) analyze collectivity in a more integrated way and (2) enables us to identify types of collectives that go beyond the obvious candidates.

In this introduction, we propose collectivity, foundationally, is about how screened-off the macroscale is from its constituents, such that in the limit of maximum screening off, no recourse to the microscale is necessary for prediction at the macroscale. Showing this, we suggest, requires meeting three conditions (see Krakauer and Flack 2010; Krakauer et al. 2011). First demonstrating that macroscopic variables are fundamental and not nominal-that is, they can be derived from the microscopic behavior of the system. Second it requires showing statistical evidence for "law like" relations among a set of macroscopic variables like mass scaling sublinearly with metabolic rate in mammals (e.g., West et al. 2002). Finally, it requires measuring how sensitive these relationships are to microscopic perturbations. We propose that, taken together, conditions 2 and 3 capture the degree to which the macroscale is screened-off from the microscale and hence its degree of collectivity. We further suggest that this relation can be formalized information theoretically (but leave the study of how for future work). An advantage of this definition is that it distinguishes collectivity from individuality, but in a comparable way: collectivity captures the information flow-the strength of the channelbetween micro and macro (for an example in the context of critical behavior see, Daniels et al. 2017), whereas individuality, formalized through the ITI as temporal uncertainty reduction (Krakauer et al. 2020), quantifies the information flow-the strength of the channel-between past and future macroscopic states of a system

The widespread debates on "how far down to go" that have re-ignited as better microscopic data have become available in neuroscience (e.g., Krakauer et al. 2017) and the study of collective behavior (e.g., Bain and Bartolo 2019), among other fields, demonstrate the need for a formal framework that can quantify on a system-by-system basis the importance of the microscale to macroscale behavior and that can account for changes in which channel dominates dynamics. Recent work that hit indirectly on the significance of such an approach comes from Muscarella and O'Dwyer (2020) who showed using an empirically grounded, coarsegrained model of a species' cellular metabolism, the surprising result that weaker competitors can be sustained in population due to properties of metabolic networks one level down. In other words, microscale behavior, usually assumed to be screened-off in species-species interactions, is in fact relevant to macroscale interactions, but to "see" this effect, the microscale behavior must be properly coarse-grained. This example illustrates that more careful thinking about how collective a system is can, in a very real way, influence model and theory building.

Beyond formalizing channel switches and detecting them, a goal should also be to understand what causes the macroscale to be screened-off. It seems likely the degree to which the macroscale is screened-off from the microscale will be influenced by network structure, the degree to which interactions among constituents are decomposable, and the complexity of the algorithms integrating information in a system to produce a collective output.

We suggest that to make progress work is needed on three fronts. The papers in this special issue largely fall into the third category.

1. Proper description of macroscale behavior, including identification of key collective modalities such as syncing and swarming and description of how they interact. This set of questions concerns the phenomenology of collectivity.

2. Development of a first principles formalism that takes into account conditions 2 and 3 above to quantify degree of macroscale collectivity. This type of research contributes to a formal theory of collectivity and the problem of measurement.

3. Study of how degree of macroscale collectivity, or screening off, is influenced by system properties like network structure, algorithms of information aggregation, and mesoscale decomposability. This of course is rife with technical problems including how to quantify algorithmic complexity and decomposability. In the latter case, there is a growing zoo of decomposability and 
synergy measures from partial information decomposition grounded in information theory to measures of variance in statistics and understanding (e.g., reviewed in Tekin 2017). This set of questions is about the mechanisms of collective behavior.

Taken together, advances on these questions will contribute to a proper theory of collective behavior.

\section{References}

Bain N, Bartolo D (2019) Dynamic response and hydrodynamics of polarized crowds. Science 4:46-49

Bialek W et al (2012) Statistical mechanics for natural flocks of birds. Proc Natl Acad Sci USA 109:4786-4791

Buhl J, Sumpter DJ, Couzin ID, Hale JJ, Despland E, Miller ER, Simpson SJ (2006) From disorder to order in marching locusts. Science 312:1402-1406

Cavagna A, Conti D, Creato C et al (2017) Dynamic scaling in natural swarms. Nat Phys 13:914-918

Daniels B, Krakauer D, Flack J (2017) Control of finite critical behaviour in a small-scale social system. Nat Commun 8:14301

Jhawar J, Guttal V (2020) Noise-induced effects in collective dynamics and inferring local interactions from data. Phil Trans R Soc B 375:20190381. https://doi.org/10.1098/rstb.2019.0381

Krakauer D, Flack J (2010) Better living through physics. Nature 467:661

Krakauer DC, Collins JP, Erwin D, Flack JC, Fontana W, Laubichler MD, Prohaska SJ, West GB, Stadler PF (2011) The challenges and scope of theoretical biology. J Theor Biol 276(1):269-276
Krakauer JW, Ghazanfar AA, Gomez-Marin A, MacIver MA, Poeppel D (2017) Neuroscience needs behavior: correcting a reductionist bias. Neuron 93:480-490

Krakauer D, Bertschinger N, Olbrich E et al (2020) The information theory of individuality. Theory Biosci 139:209-223

Mora T, Walczak AM, Bialek W, Callan CG (2010) Maximum entropy models for antibody diversity. Proc Natl Acad Sci 107:5405-5410

Muscarella ME, O'Dwyer JP (2020) Species dynamics and interactions via metabolically informed consumer-resource models. Theor Ecol 13:503-518

O'Keeffe KP, Hong H, Strogatz SH (2017) Oscillators that sync and swarm. Nat Commun 8:1504

Ramírez-Ávila GM, Kurths J, Depickère S, Deneubourg J-L (2019) Modeling fireflies synchronization. Springer, New York, pp 131-156

Sarfati R, Hayes Julie C, Peleg O (2021) Self-organization in natural swarms of Photinus carolinus synchronous fireflies. Sci Adv 7. https://doi.org/10.1126/sciadv.abg9259

Tekin E, Savage VM, Yeh PJ (2017) Measuring higher-order drug interactions: a review of recent approaches. Curr Opin Syst Biol 4:16-23

West GB, Woodruff WH, Brown JH (2002) Allometric scaling of metabolic rate from molecules and mitochondria to cells and mammals. Proc Natl Acad Sci 99(suppl 1):2473-2478

Publisher's Note Springer Nature remains neutral with regard to jurisdictional claims in published maps and institutional affiliations. 